\title{
Impact of antithrombotics on the fecal immunochemical test for colorectal can- cer screening : a multi-center Belgian experience
}

\author{
L. Wauters ${ }^{1}$, V.R.H. Van der Voort ${ }^{2}$, P. Dobbels ${ }^{3}$, K. Hendrickx ${ }^{3}$, V. Casneuf ${ }^{3,4}$, J. Vandervoort ${ }^{3}$ \\ (1) Department of Gastroenterology and Hepatology, UZ Leuven, Leuven, Belgium and Translational Research Center for Gastrointestinal Disorders (TARGID), KU \\ Leuven, Leuven, Belgium ; (2) Department of Gastroenterology, UMC Utrecht, Utrecht, The Netherlands ; (3) Department of Gastroenterology, OLV Hospitals Aalst/Asse/ \\ Ninove, Aalst, Belgium ; (4) Department of Gastroenterology, UZ Gent, Gent, Belgium.
}

\begin{abstract}
Background: Impact of antithrombotics on the fecal immunochemical test (FIT) for colorectal cancer (CRC) screening remains unclear.

Methods : Patients undergoing colonoscopy for positive FIT in 2015 were assessed at 3 Belgian centers. Significant findings were advanced polyps (AP) (sessile serrated, tubular or villous adenomas $>1 \mathrm{~cm}$ or high-grade dysplasia) and CRC. False positive FIT and detection of AP/CRC with antithrombotics were calculated.

Results : 510 patients (64\% male, median (IQR) age 63.2 (60.266.4) years) were included. Colorectal pathology in 371/510 (73\%) was associated with male gender $(70 \%$ vs. $48 \% ; p=.0001)$ and family history ( $16 \%$ vs. $8 \% ; p=.02)$. Antithrombotics in $125 / 510$ $(25 \%)$ were associated with male gender $(78 \%$ vs. $59 \% ; p=.0001)$, older age $(65.2(62.2-70.3)$ vs. $62.3(58.7-66.3)$ years $; p=.0001)$ and GI-symptoms $(18 \%$ vs. $11 \% ; p=.04)$. False positive FIT $(25 \%$ vs. $28 \% ; p=.52)$ and detection of AP $(42 \%$ vs. $36 \% ; p=.27)$ or CRC $(6 \%$ vs. $5 \% ; p=.69)$ were similar in patients with vs. no antithrombotics. Use of antithrombotics did not predict a higher chance of colorectal pathology after adjusting for confounders.

Conclusion : Although antithrombotics were prescribed more frequently in male and older patients, detection of AP/CRC was similar. Despite increased GI symptoms, false positive FIT was similar with antithrombotics. (Acta gastroenterol. belg., 2021, 84, 19-24).
\end{abstract}

Keywords : Colon cancer, fecal immunochemical test, antithrombotics, aspirin, screening

Abbreviations : AP, advanced polyp ; CI, confidence interval ; CRC, colorectal cancer ; GP, general practitioner ; FAP, familial adenomatous polyposis ; FIT, fecal immunochemical test ; GI, gastrointestinal; HNPCC, hereditary non-polyposis colon cancer; IBD, inflammatory bowel disease ; IQR, interquartile range ; NSAID, non-steroidal anti-inflammatory drugs; OR, odds ratio ; PPV, positive predictive value ; RBPA, red blood loss per anum; SSA/P, sessile serrated adenoma/polyp, TSA; traditional serrated adenoma.

\section{Introduction}

Programmatic screening for colorectal cancer (CRC) in Flanders (Belgium) has been implemented since October 2013 with the fecal immunochemical test (FIT) for adults aged between 56 and 74 years (1). This method of screening is now widely recommended throughout Europe (2). While screening is intended for asymptomatic individuals in the general population, patients with gastrointestinal (GI) symptoms and/or a positive family history of CRC should undergo colonoscopy immediately. However, in clinical practice many of these patients often undergo FIT ordered by the general practitioner (GP) first with subsequent referral for colonoscopy if positive (3). This 'opportunistic' screening (also in patients younger than 56 years of age) may influence the results of FIT due to different pre-test probability and this group of patients has been excluded in previous studies, including only the heavily studied patient population from screening programs.

The impact of antithrombotics on the performance of FIT for CRC screening in the general population remains unclear. Antithrombotics include both antiplatelet agents and anticoagulants and previous studies recommended against stopping these drugs before FIT (4-7). Moreover, as these drugs lead to a higher incidence of GI bleeding, the detection of advanced neoplasia might even be increased with use of antithrombotics prior to FIT $(7,8)$. Real-life experience on the impact of all the possible antihrombotics (both antiplatelet or anticoagulant drugs) and aspirin alone in the general population with both programmatic or opportunistic screening prior to FIT for CRC screening is scarce.

We analyzed a prospective cohort of adults undergoing colonoscopy for positive FIT between January and December 2015 in Flanders. Firstly, we aimed to compare the use of antithrombotics or aspirin alone in patients with or without colorectal pathology and significant findings. Secondly, we aimed to assess the rates of false positive FIT and detection of polyps and CRC in patients with and without antithrombotics or aspirin alone. We also compared the diagnostic performance of FIT through programmatic or opportunistic CRC screening, in order to rule out a differential effect in different patient populations. Finally, multivariate analysis was performed to assess the effect of antithrombotics or aspirin alone on colorectal pathology and significant findings, adjusting for age, sex, family history of CRC and GI-symptoms.

Correspondence to : Lucas Wauters, Department of Gastroenterology and Hepatology, UZ Leuven, Leuven, Belgium. Tel. 003216372093, Fax. 003216330723 .

Email: lucas.wauters@kuleuven.be

Submission date : 10/05/2020

Acceptance date : 21/05/2020 


\section{Materials and methods}

\section{Patient population}

Patient data on all adults (aged $>40$ years old) undergoing colonoscopy for positive FIT through programmatic or opportunistic CRC screening between January and December 2015 were prospectively collected at 3 affiliated centers (OLV Aalst, Asse and Ninove in Flanders, Belgium). Exclusion criteria included personal history of inflammatory bowel disease (IBD), GI-surgery (gastric, ileocecal, colonic or anal) and personal or family history of CRC, including familial adenomatous polyposis (FAP) and hereditary non-polyposis colon cancer (HNPCC). Current use of antithrombotics at the time of endoscopy was systematically documented on admission records. Patient gender, age at colonoscopy, GI-symptoms (including red blood loss per anum (RBPA), melena, change in bowel habits or anemia) and family history of polyps or CRC were noted at the time of endoscopy or assessed through review of medical records. The study was approved by the ethics committee of the OLV Hospitals Aalst/Asse/Ninove (2017/089).

\section{CRC screening}

Programmatic screening for $\mathrm{CRC}$ in Flanders (Belgium) is organized through the "Bevolkingsonderzoek Dikkedarmkanker", targeting all men and women living in Flanders aged between 56 and 74 years. In 2015, a personal invitation was sent out by mail to all adults with paired ages (56 years, 58, 60, etc.). Patients with present or previous CRC ( $<10$ years), colectomy, prior colonoscopy or FIT in the previous two years were automatically excluded from the program through exclusion lists, drawn up by the "Stichting Kankerregister", which are automatically coupled with the database of the centre for cancer detection four times a year.

The sampling kit consists of the FIT (OC Sensor DIANA, Eiken Chemical, Japan) with the necessary material and instructions for taking the sample and returning by mail to a centralized laboratory (CMA, Herentals, Belgium) free of charge. Using a cut-off value of $75 \mathrm{ng} / \mathrm{ml}$, the result is sent to the patient and their GP within 14 days. A positive test should be followed by a colonoscopy, ordered either through the GP or by consulting a gastroenterologist. In case of a negative result, an invitation for a repeat test is sent every 2 years, unless the patient is excluded due to the above mentioned reasons or the patient wishes to withdraw via a response form.

\section{Colorectal pathology}

Endoscopy and histopathology reports were crosschecked for the presence of colorectal pathology. Normal endoscopic findings also included uncomplicated diverticulosis or hemorrhoids. Benign findings were angio- dysplasia and hyperplastic or inflammatory polyps. Premalignant polyps were defined as tubular adenomas measuring $<1 \mathrm{~cm}$ with low-grade dysplasia. Missing histology or failure to retrieve polyps was only included if endoscopic appearance showed a sessile polyp $<1$ $\mathrm{cm}$ which was considered potentially premalignant. Significant findings were defined as advanced polyps (AP), including sessile serrated adenomas/polyps (SSA/P) and tubular, tubulovillous or villous adenomas measuring $>1 \mathrm{~cm}$ or with high-grade dysplasia, and malignant polyps or CRC in case of submucosal invasion.

\section{Antithrombotics}

Antithrombotic drugs included the antiplatelet agents acetyl salicyclic acid or aspirin 80mg O.D., the thienopyridine clopidogrel $75 \mathrm{mg}$ O.D., dipyridamole $200 \mathrm{mg}$ B.I.D. and ticagrelor 60-90mg O.D. as well as anticoagulant drugs, both the direct oral anticoagulants (rivaroxaban, dabigatran and apixaban - dosage depending on indication, age and kidney function) and vitamin $\mathrm{K}$ antagonists (warfarin, phenprocoumon and acenocoumarol - dosage based on the International Normalized Ratio (INR)). Aspirin was used either in combination with other antiplatelet or anticoagulant drugs or alone in standard dosing of $80 \mathrm{mg}$ O.D. depending on the indication. Cessation of antithrombotics was not recommended prior to fecal sampling for FIT, as advised by the European guidelines(9).

\section{Statistical analysis}

Categorical data were summarized as percentages and continuous data as medians plus interquartile ranges (IQR). Categorical data were analyzed using $\chi^{2}$ tests and continuous data using Mann-Whitney U tests. Univariate analyses were performed between patients with and without colorectal pathology or significant findings and between patients with and without antithrombotics or aspirine alone using Prism GraphPad Software (La Jolla, CA, USA). Multivariate analysis was performed with SPSS Statistics for Windows (Chicago, IL, USA). A binary logistic regression model was made with calculation of odds ratio's (OR) and 95\% confidence intervals (CI) for finding colorectal pathology or significant findings with the use of antithrombotics and aspirin alone, adjusted for sex, age, family history of CRC and GI-symptoms.

\section{Results}

\section{Patient selection}

Demographic and clinical data were collected from a total of 531 patients undergoing colonoscopy for positive FIT in 2015, of which 1 with polyposis syndrome, 3 with IBD and 15 with GI-surgery were excluded. In addition, 2 patients with missing data were excluded, resulting in a total of 510 patients ( $64 \%$ male, median (IQR) age 


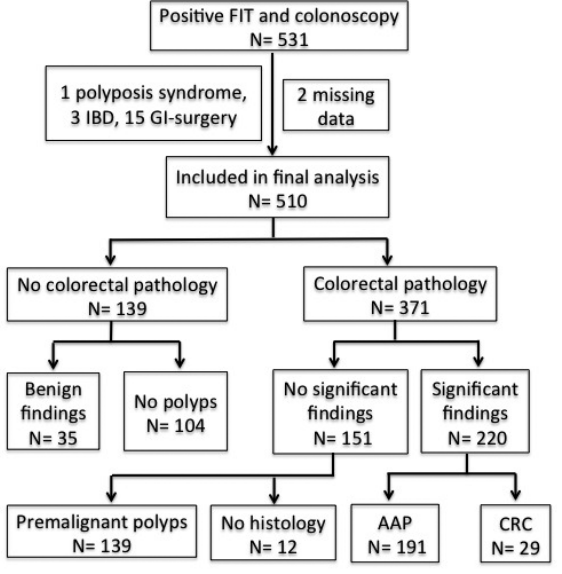

Figure 1. - Patient flow chart for the present study.

63.2 (60.2-66.4) years) with positive FIT (Figure 1). Endoscopy and histopathology reported only benign findings in 35/510 (7\%) patients and endoscopy was reported as normal in 104/510 (20\%) patients. The rate of false-positive FIT in the study population was therefore 139/510 (27\%).

\section{Colorectal pathology}

Colorectal pathology was confirmed in 371/510 (73\%) patients, of which 139/371 (37\%) premalignant polyps and $12 / 371(3 \%)$ missing histology $(27 \%$ and $2 \%$ of the total cohort, resp.) (Figure 1). Colorectal pathology was associated with male gender $(70 \%$ vs. $48 \% ; \mathrm{p}=$ $.0001)$ and with positive family history $(16 \%$ vs. $8 \% ; p=$ .02 ) compared to patients with no colorectal pathology $(n=139)($ Table 1$)$. There were no differences in GI symptoms, use of antithrombotics or aspirin alone in patients with or without colorectal pathology. Significant findings were found in 220/371 (59\%) patients with colorectal pathology ( $43 \%$ of the total cohort), of which 191/220 (87\%) were AP and 29/220 (13\%) CRC (37\% and $6 \%$ of the total cohort, resp.) (Figure 1). No significant differences were found in demographic or clinical variables (including antithrombotics or aspirin alone) between patients with $(n=220)$ or without $(n=$ 151) significant findings (Table 1$)$.

\section{Antithrombotics}

Antithrombotic drugs were prescribed in $125 / 510$ $(25 \%)$ and aspirin alone in $87 / 510(17 \%)$ patients. Others included clopidogrel in 10 cases, dipyridamole in 3, ticagrelor in 2, direct oral anticoagulants in 13 and vitamin $\mathrm{K}$ antagonists in 10 (with overlap). Antithrombotics were associated with male gender (78\% vs. $59 \% ; \mathrm{p}=.0001)$, older age (65.2 (62.2-70.3) vs. $62.3(58.7-66.3)$ years ; $\mathrm{p}=.0001)$ and GI symptoms $(18 \%$ vs. $11 \% ; \mathrm{p}=.04)$ (Table 2). Aspirin alone was also associated with male gender $(80 \%$ vs. $59 \% ; \mathrm{p}=.0002)$ and older age $(64.4$ (61.1-69.6) vs. $62.3(58.7-66.3)$ years ; $p=.0003)$. Rates of false positive FIT ( $25 \%$ vs. $28 \% ; \mathrm{p}=.52)$, detection of $\operatorname{AP}(42 \%$ vs. $36 \% ; p=.27)$ and CRC (6\% vs. $5 \% ; p=.69)$ were similar in patients with or without antithrombotics $(\mathrm{n}=385)$. Rates of false positive FIT $(26 \%$ vs. $28 \%$; $\mathrm{p}=$ $.79)$ and detection of AP (41\% vs. $36 \% ; p=.36)$ and CRC (7\% vs. $5 \% ; \mathrm{p}=.60)$ were similar in patients with aspirin alone compared to no antihrombotics (Table 2).

Table 1. - Comparison of variables of interest between patients with $(n=371)$ and without $(n=139)$ colorectal pathology and in the former for subjects with $(n=220)$ and without $(n=151)$ significant findings

\begin{tabular}{|l|c|c|c|c|c|c|}
\hline & $\begin{array}{c}\text { Colorectal pathology } \\
(\mathrm{n}=371)\end{array}$ & $\begin{array}{c}\text { No colorectal pathology } \\
(\mathrm{n}=139)\end{array}$ & P-value & $\begin{array}{c}\text { Significant findings } \\
(\mathrm{n}=220)\end{array}$ & $\begin{array}{c}\text { No significant findings } \\
(\mathrm{n}=151)\end{array}$ & P-value \\
\hline Male (\%) & $258(70)$ & $66(47)$ & $\mathbf{. 0 0 0 1}$ & $152(69)$ & $106(70)$ & .81 \\
\hline Median (IQR) age in years & $64(60-66)$ & $62(58-66)$ & .26 & $64.3(60.3-66.4)$ & $62.3(58.8-66.4)$ & .10 \\
\hline GI symptoms (\%) & $52(14)$ & $12(9)$ & .10 & $34(15)$ & $18(12)$ & .34 \\
\hline Family history (\%) & $59(16)$ & $11(8)$ & $\mathbf{. 0 2}$ & $34(15)$ & $25(17)$ & .77 \\
\hline Antithrombotics (\%) & $94(25)$ & $31(22)$ & .48 & $60(27)$ & $34(23)$ & .30 \\
\hline Aspirin alone (\%) & $64(17)$ & $23(17)$ & .58 & $42(19)$ & $22(15)$ & .17 \\
\hline
\end{tabular}

*Univariate analysis $\left(\chi^{2}\right.$-test categorical data, Mann-Whitney-U continuous data).

Table 2. - Comparison of variables of interest between patients with antithrombotics $(\mathbf{n}=\mathbf{1 2 5})$ or Aspirin alone $(\mathbf{n}=\mathbf{8 7})$ and patients without any antithrombotic $(n=385)$

\begin{tabular}{|c|c|c|c|c|c|c|}
\hline & $\begin{array}{l}\text { Anti-thrombotics } \\
(n=125)\end{array}$ & $\begin{array}{l}\text { No anti-thrombotics } \\
(\mathrm{n}=385)\end{array}$ & $\mathrm{P}$-value & $\begin{array}{c}\text { Aspirin alone } \\
(\mathrm{n}=87)\end{array}$ & $\begin{array}{l}\text { No anti-thrombotics } \\
(\mathrm{n}=385)\end{array}$ & P-value \\
\hline Male $(\%)$ & $98(78)$ & $226(59)$ & .0001 & $70(80)$ & $226(59)$ & .0002 \\
\hline Median (IQR) age in years & $65.2(62.2-70.3)$ & $62.3(58.7-66.3)$ & .0001 & $64.4(61.1-69.9)$ & $62.3(58.7-66.3)$ & .0003 \\
\hline GI symptoms (\%) & $22(18)$ & $42(11)$ & .04 & $10(11)$ & $42(11)$ & .87 \\
\hline Family history (\%) & $15(12)$ & $55(14)$ & .52 & $11(13)$ & $55(14)$ & .69 \\
\hline False positive FIT (\%) & $31(25)$ & $107(28)$ & .52 & $23(26)$ & $107(28)$ & .79 \\
\hline Detection rate of AP $(\%)$ & $52(42)$ & $139(36)$ & .27 & $36(41)$ & $139(36)$ & .36 \\
\hline Detection rate of CRC (\%) & $8(6)$ & $21(5)$ & .69 & $6(7)$ & $21(5)$ & .60 \\
\hline
\end{tabular}

*Univariate analysis ( $\chi^{2}$-test categorical data, Mann-Whitney-U continuous data). 
Table 3. - Comparison of variables of interest between patients with positive FIT through programmatic $(n=463)$ or opportunistic screening $(n=47)$

\begin{tabular}{|l|c|c|c|}
\hline & Opportunistic screening $(\mathrm{n}=47)$ & Programmatic screening $(\mathrm{n}=463)$ & P-value \\
\hline Male (\%) & $32(68)$ & $292(63)$ & .50 \\
\hline Median (IQR) age in years & $64.4(58.1-72.1)$ & $62.9(60.2-66.4)$ & .54 \\
\hline GI symptoms (\%) & $10(21)$ & $54(12)$ & .06 \\
\hline Family history (\%) & $3(6)$ & $67(14)$ & .12 \\
\hline Antithrombotics (\%) & $14(30)$ & $711(24)$ & .38 \\
\hline Aspirin alone (\%) & $12(26)$ & $124(27)$ & .85 \\
\hline False positive FIT (\%) & $15(32)$ & $177(38)$ & .45 \\
\hline Detection rate of AP (\%) & $14(30)$ & $27(6)$ & .25 \\
\hline Detection rate of CRC (\%) & $2(4)$ & .62 \\
\hline
\end{tabular}

*Univariate analysis ( $\chi^{2}$-test categorical data, Mann-Whitney-U continuous data).

\section{CRC screening}

There were no differences in demographics between patients with programmatic $(n=463)$ or opportunistic $(n=47)$ screening (Table 3$)$. Although the presence of GI symptoms is higher in patients with opportunistic (21\%) compared to programmatic screening (12\%), the difference was not statistically significant. Positive family history and the use of antithrombotics or aspirin alone were as common. The rates of false positive FIT (32\% vs. $27 \% ; p=.45)$, detection of AP (30\% vs. $38 \%$; $\mathrm{p}=.25)$ and CRC (4\% vs. $6 \% ; \mathrm{p}=.62)$ were also similar in both groups.

\section{Multivariate analysis}

After adjusting for sex, age, family history of CRC and GI-symptoms, the use of antithrombotics (OR 0.88, $95 \% \mathrm{CI}=0.53-1.45 ; \mathrm{p}=.61$ ) or aspirin alone (OR 0.81, $95 \% \mathrm{CI}=0.45-1.41 ; \mathrm{p}=.45)$ did not independently predict a higher chance of finding colorectal pathology on colonoscopy. The use of antithrombotics (OR 1.05, $95 \% \mathrm{CI}=0.68-1.62 ; \mathrm{p}=.81$ ) or aspirin alone (OR 1.13, $95 \% \mathrm{CI}=0.70-1.81, \mathrm{p}=.63)$ did not independently predict a higher chance of significant findings (CRC and AP), after adjusting for age, sex, family history of CRC and GI-symptoms.

\section{Discussion}

In this prospective cohort, the impact of antithrombotics on FIT testing was studied by assessing the rate of false positive FIT and the detection of colorectal pathology and significant findings in adults aged $>40$ years, with or without routine CRC screening indications such as presence of GI symptoms or positive family history. Patients undergoing endoscopy for positive FIT through either programmatic or opportunistic CRC screening were included in order to reflect real-life practice with no differences between both screening modalities. False positive FIT (including benign findings and normal endoscopy) occurred in $27 \%$ of patients and colorectal pathology was present in $73 \%$ with significant findings in $43 \%$ of patients. Detection rate of AP was $37 \%$ and of CRC 6\% for the whole cohort. Antithrombotics (including aspirin alone) were prescribed in $25 \%$ of patients and more often in male and older patients with an inherent higher cancer risk. However, detection rates of AP and CRC were similar when compared to patients with no antithrombotics. Antithrombotics were associated with a higher rate of GI symptoms, which did however not increase the rate of false positive FIT. After adjusting for possible confounders, multivariate analysis showed antithrombotics and aspirin alone did not independently predict a higher chance of finding colorectal pathology or significant findings. Therefore, there seems to be no impact of the use of antithrombotics on the diagnostic performance of FIT for CRC screening in the general population in Flanders.

Programmatic CRC screening with FIT (OC Sensor) in Flanders is sent out every two years to adults between 56 and 74 years old. While only patients with paired ages were invited for programmatic screening in 2015, there is also overlap with patients invited in 2014 but undergoing colonoscopy only after January 2015. Patients with previous CRC $(<10$ years $)$ and screening with colonoscopy or FIT ( $<2$ years) are automatically excluded from the screening program. In clinical practice, patients with GI symptoms or positive family history may have a FIT ordered via the GP before undergoing colonoscopy. This also applies to patients between 40 and 55 years old, as they are not (yet) included in the screening program. Although the presence of GI symptoms was higher in patients with opportunistic compared to programmatic screening, this was probably higher as we did not systematically assess symptoms. Nevertheless, a strength of this study is the inclusion of both groups, reflecting real life practice and revealing no significant differences in both groups in terms of diagnostic performance of FIT.

Although reduction in mortality by CRC screening has been demonstrated with the fecal occult blood test Hemoccult, this is a guaiac-based test (10). FIT is preferred due to its higher sensitivity and specificity with less false positive results due to upper GI bleeding because FIT only reacts to globin, which is degraded by proteases along the GI tract $(8,11)$. In contrast to 
studies with Hemoccult showing decreased positive predictive value (PPV) for advanced neoplasia (12), FIT showed a higher sensitivity with similar or slightly lower specificity $(4,8)$ and PPV (5-7) for detecting AP and CRC with use of antithrombotics. However, a meta-analysis found similar PPV of CRC with FIT in patients taking aspirin but increased PPV with warfarin (13). In contrast, the anticoagulant acenocoumarol alone or in combination with aspirin or clopidogrel increased FIT positivity with a similar PPV when using the OC Sensor with a cut-off of $75 \mathrm{ng} / \mathrm{ml}$, which is the same as in the Flemish screening program (7).

Antithrombotics are associated with GI bleeding, which was also evident from the higher incidence of GI symptoms, including RBPA and melena. Anemia was reported in a minority of patients, wherefore further investigation with gastroscopy was not necessary as even iron deficiency anemia may be seen with occult bleeding from the proximal colon. However, aspirin alone in standard dosing is not associated with clinically important GI-bleeding, as reflected in the similar rate of GI symptoms. Its use mostly leads to upper GI-bleeding (14), which is not detected by FIT due to selective reactivity to hemoglobin and explaining the similar rate of false positive FIT. Bleeding from lower GI-sources other than CRC, such as benign or potentially premalignant lesions would not necessarily increase their detection, as the bleeding may be intermittent or dispersed throughout the sample and quantity depends on the size. Some have even advocated that the detection of advanced neoplasia might be increased with the use of antithrombotics prior to FIT $(7,8)$. We also found no difference in GI symptoms between patients with and without colorectal pathology, as the latter group also includes diverticulosis or hemorrhoids and angiodysplasia or inflammatory polyps, which may also cause GI symptoms.

The detection rate of AP (37\%) in the present study is higher compared to previous studies and may be due to the inclusion of SSA/P of $>1 \mathrm{~cm}$ or with dysplasia, which have not been consistently reported and for which cold snare polypectomy is also feasible(15). Serrated lesions, including hyperplastic polyps, SSA/P with or without dysplasia and traditional serrated adenomas (TSA) are often flat and covered with mucus and difficult to visualize during colonoscopy. SSA/Ps have been identified as main precursors of CRC and it was estimated that $\geq$ $15 \%$ of CRCs might arise through the serrated neoplasia pathway (16). TSAs are also considered premalignant, but the prevalence of these lesions is low. There were only 7 advanced SSA/P identified in the present cohort, whereas hyperplastic polyps were considered as benign findings, especially those of $<1 \mathrm{~cm}$ and located in the rectosigmoid colon. The detection rate of CRC $(6 \%)$ and false positive FIT $(27 \%)$ is comparable with previous studies in Flanders (17).

The strength of the present study is the homogenous inclusion of all patients undergoing colonoscopy at 3 affiliated centers in Flanders for positive FIT, either through programmatic or opportunistic screening. Data on clinical variables including use of antithrombotics was available through questioning at the time of endoscopy and review of medical records. All endoscopic and histologic examinations were uniformly performed at affiliated centers, where quality is overseen by accreditation. The limitations are the relative small number of patients recruited only during 2015 and the absence of data on patients with colonoscopy after negative FIT, which prevented further subgrouping of significant findings according to location or subtype and calculating sensitivity or negative predictive value. We did not have information on cardiovascular or CRC risk factors, such as diet or smoking, which are difficult to correctly assess due to the cumulative effect in time. Nonsteroidal anti-inflammatory drugs (NSAIDs) were also not included, as these are often used only intermittently. Diverticulosis or hemorrhoids were not excluded from the analysis, as these findings are common and not always routinely reported in endoscopy reports. Missing histology or failure to retrieve small polyps was included in the group with colorectal pathology, as they were potentially premalignant with further endoscopic followup depending on the endoscopic appearance. Although endoscopy was performed in all patients, some polyps might have been missed as previously demonstrated by tandem colonoscopy (18). Use of antithrombotics and aspirin was related to age and gender, which might affect test performance (19). However, after adjusting for these possible confounders in a multivariate analysis, the results were unchanged.

In conclusion, this prospective Flemish cohort found no impact of antithrombotics or aspirin alone on the performance of FIT for CRC in the general population. Although antithrombotics (including aspirin alone) were highly prescribed in male and older patients with an inherent higher cancer risk, detection rates of AP and $\mathrm{CRC}$ were not increased. Despite the higher rate of GI symptoms with antithrombotic drugs, this did not lead to more false positive FIT. We therefore conclude that there seems to be no reason to discontinue antithrombotics during screening with FIT.

\section{References}

\footnotetext{
1. Bevolkingsonderzoek Dikkedarmkanker. https://dikkedarmkanker. bevolkingsonderzoek.be/

2. STOCK C, BRENNER H. Utilization of lower gastrointestinal endoscopy and fecal occult blood test in 11 European countries : evidence from the Survey of Health, Aging and Retirement in Europe. Endoscopy, 2017, 42(7) : 546-56.

3. STONESTREET J, CHANDRAPALAN S, WOOLLEY D, UTHMAN U, ARASARADNAM RP. Systematic Review and Meta-Analysis : Diagnostic Accuracy of Faecal Immunochemical Testing for Haemoglobin (FIT) in Detecting Colorectal Cancer for Both Symptomatic and Screening Population. Acta Gastroenterol. Belg., 2019, 82(2) : 291-9.

4. LEVI Z, ROZEN P, HAZAZI R, VILKIN A, WAKED A, MAOZ E, et al. Sensitivity, but Not Specificity, of a Quantitative Immunochemical Fecal Occult Blood Test for Neoplasia Is Slightly Increased by the Use of LowDose Aspirin, NSAIDS, and Anticoagulants. Am. J. Gastroenterol., 2009, 104(4) : 933-8.
} 
5. MANDELli G, RADAELli F, PAGGI S, TERRENI N, GOLA G, GRAMEGNA M, et al. Anticoagulant or aspirin treatment does not affect the positive predictive value of an immunological fecal occult blood test in patients undergoing colorectal cancer screening. Eur. J. Gastroenterol. Hepatol., 2011, 23(4) : 323-6.

6. TSUJI Y, GUNJI T, SATO H, ONO A, ITO T, OHATA K, et al. Antithrombotic drug does not affect the positive predictive value of an immunochemical fecal occult blood test. Dig. Endosc., 2014, 26(3) : 424-9.

7. BUJANDA L, SARASQUETA C, LANAS Á, QUINTERO E, CUBIELLA J, HERNANDEZ V, et al. Effect of oral anticoagulants on the outcome of faecal immunochemical test. Br. J. Cancer, 2014, 110(5) : 1334-7.

8. BRENNER H, TAO S, HAUG U. Low-Dose Aspirin Use and Performance of Immunochemical Fecal Occult Blood Tests. JAMA, 2010, 304(22) : 2513.

9. HALLORAN SP, LAUNOY G, ZAPPA M. European guidelines for quality assurance in colorectal cancer screening and diagnosis. First Edition Faecal occult blood testing. Endoscopy, 2012, 44 : suppl. 3.

10. HEWITSON P, GLASZIOU P, WATSON E, TOWLER B, IRWIG L. Cochrane systematic review of colorectal cancer screening using the fecal occult blood test (hemoccult) : an update. Am. J. Gastroenterol., 2008, 103(6) : 1541-9.

11. PARK D IL, RYU S, KIM Y-H, LEE S-H, LEE CK, EUN CS, et al. Comparison of guaiac-based and quantitative immunochemical fecal occult blood testing in a population at average risk undergoing colorectal cancer screening. Am. J. Gastroenterol., 2010, 105(9) : 2017-25.
12. SAWHNEY MS, MCDOUGALL H, NELSON DB, BOND JH. Fecal occult blood test in patients on low-dose aspirin, warfarin, clopidogrel, or nonsteroidal anti-inflammatory drugs. Dig Dis Sci 2010, 55(6) : 1637-42.

13. GANDHI S, NARULA N, GANDHI S, MARSHALL JK, FARKOUH ME. Does acetylsalicylic acid or warfarin affect the accuracy of fecal occult blood tests? J Gastroenterol Hepatol 2013, 28(6) : 931-6.

14. CAMPBELL CL, SMYTH S, MONTALESCOT G, STEINHUBL SR. Aspirin Dose for the Prevention of Cardiovascular Disease. JAMA, 2007, 297(18) : 2018.

15. VAN OVERBEKE L, ILEGEMS S, MERTENS G, MORTIER L, VAN DONGEN K, VERBEKE L, et al. Cold Snare Endoscopic Resection of Nonpedunculated Colorectal Polyps Larger Than $10 \mathrm{Mm}$. A Retrospective Series. Acta Gastroenterol. Belg., 2019, 82(4) : 475-8.

16. IJSPEERT JEG, VERMEULEN L, MEIJER GA, DEKKER E. Serrated neoplasia-role in colorectal carcinogenesis and clinical implications. Nat. Rev. Gastroenterol. Hepatol., 2015, 12(7) : 401-9.

17. COPPENS G, GEBOES K, DE LOOZE D, HINDRYCKX P, DE VOS M. Screeningsonderzoek naar darmkanker in vlaanderen met immunochemische fecaal-occultbloedtest. Tijdschr. Genees., 2016, 72(20) : 1169-75.

18. VAN RIJN JC, REITSMA JB, STOKER J, BOSSUYT PM, VAN DEVENTER SJ, DEKKER E. Polyp miss rate determined by tandem colonoscopy : a systematic review. Am. J. Gastroenterol., 2006, 101(2) : 343-50.

19. BRENNER H, HAUG U, HUNDT S. Sex Differences in Performance of Fecal Occult Blood Testing. Am. J. Gastroenterol., 2010, 105(11) : 2457-64. 